\title{
Effects of the Digital Game "Fit, Food, Fun" on Nutritional Knowledge: A Pilot Study among German Children and Adolescents
}

\author{
Sophie Laura Holzmann ${ }^{1}$, Hanna Schaefer ${ }^{2}$, Georg Groh $^{2}$, David Alexander Plecher ${ }^{3}$, \\ Gudrun Klinker ${ }^{3}$, Gunther Schauberger ${ }^{4}$, Hans Hauner ${ }^{1,5}$ and Christina Holzapfel ${ }^{1}$ \\ ${ }^{1}$ Institute for Nutritional Medicine, Else Kroener-Fresenius-Center for Nutritional Medicine, School of Medicine, \\ Klinikum rechts der Isar, Technical University of Munich, Munich, Germany, \\ ${ }^{2}$ Research Group Social Computing, Department of Informatics, Technical University of Munich, Garching, Germany, \\ ${ }^{3}$ Chair for Computer Aided Medical Procedures \& Augmented Reality, Department of Informatics, Technical \\ University of Munich, Garching, Germany, \\ ${ }^{4}$ Chair of Epidemiology, Department of Sport and Health Sciences, Technical University of Munich, Munich, Germany \\ and \\ ${ }^{5}$ ZIEL-Institute for Food and Health, Nutritional Medicine Unit, Technical University of Munich, Freising, Germany
}

\begin{abstract}
Introduction: Serious games are a novel and entertaining approach for digital health education in the younger population. Aim of this pilot study was to evaluate the short-term effectiveness of the serious game "Fit, Food, Fun" (FFF) among a subset of children and adolescents in Germany with regard to nutritional knowledge.
\end{abstract}

Materials and Methods: Two Bavarian secondary schools were each allocated to one intervention arm. The gameplay intervention (gameplay group; GG) consisted of a 15-minute gameplay session for each of three days (Tuesday to Thursday), while the teaching intervention (teaching group; TG) was performed as a 15-minute classic lecture for the same number of days. Given nutritional information was based on the recommendations of the "German Nutrition Society e.V." and was identical for both intervention groups. Nutritional knowledge was evaluated through a standardised questionnaire at baseline (Monday) and post-intervention (Friday). Lifestyle behaviour (diet; physical activity) and anthropometrics (height; weight) were assessed once at baseline. Inclusion criteria were sufficient German language skills and parental consent form. Statistical analyses were performed using the statistical software R (R Core Team, 2018).

Results: In total, 47 students ( $62 \%$ male) were assigned to the GG and 47 students $(72 \%$ male) to the TG. The mean age was 13.5 years in the GG and 12.8 years in the TG. The mean body mass index was in the normal range (GG: $\left.24.4 \mathrm{~kg} / \mathrm{m}^{2} ; \mathrm{TG}: 22.0 \mathrm{~kg} / \mathrm{m}^{2}\right)$. Data at baseline and post-intervention are analysed for 36 participants in the GG and for 40 participants in the TG. Compared to baseline, results revealed significant improvements ( $\mathrm{p}$-value $<0.001$ ) in nutritional knowledge in both intervention groups. There was a between-group difference with significantly ( $p$-value $=0.0139)$ higher increase of nutritional knowledge in the TG.

Discussion: This pilot study provides evidence for the short-term efficacy of both game-based and traditional education approaches on the improvement of nutritional knowledge; however, further research in warranted to assess the potential effect of a digital gameplay intervention on nutritional behaviour. Finally, the FFF game might be considered as an appropriate educational tool for imparting nutritional knowledge in an entertaining and effective format among children and adolescents.

\section{Conflict of Interest}

There is no conflict of interest. 